\title{
Subgrid parameterisation with scaling laws for atmospheric and oceanic flows
}

\author{
$\underline{\text { V. Kitsios }}^{\text {a }}$, J.S. Frederiksen ${ }^{\text {a }}$, M.J. Zidikheri ${ }^{\text {b }}$ \\ ${ }^{a}$ Centre for Australian Weather and Climate Research, CSIRO Marine and Atmospheric Research, \\ Aspendale, Victoria, Australia \\ ${ }^{\mathrm{b}}$ Centre for Australian Weather and Climate Research, Bureau of Meteorology, Melbourne, Victoria, \\ Australia \\ Email: vassili.kitsios@csiro.au
}

\begin{abstract}
:
Subgrid-scale (SGS) parameterisations of turbulence with self similar scaling laws are developed for large eddy simulations (LESs) of atmospheric and oceanic fows. The stochastic SGS modelling approach of Frederiksen and Kepert (2006) is used to determine the model coeff cients self-consistently from higher resolution reference direct numerical simulation (DNS). The resulting LES then replicates the statistics of the DNS at the resolved scales. In general the SGS model coeff cients depend on both the zonal and total wavenumbers, making them anisotropic.

The fow felds are simulated using a two-level quasi-geostrophic model that incorporates the processes of baroclinic instability and the interaction of synoptic-scale structures and inhomogeneous Rossby wave turbulence. Two specif c basic fows are analysed: an atmospheric fow with large scale jets in the midlatitudes; and an oceanic fow representative of the Antarctic Circumpolar Current. Despite the obvious differences, these fow f elds exhibit similar turbulent properties. In both cases the turbulent energy in the system is injected at the Rossby wavenumber $\left(k_{R}\right)$, and there is a constant transfer of enstrophy from the Rossby waves to the small-scale (high wavenumber) structures. The key difference, is that in the present simulations the baroclinically unstable Rossby waves within the atmosphere occur at $k_{R} \approx 14$, whilst in the ocean $k_{R} \approx 140$. This makes the ocean a more computationally challenging case, as a f ner grid is required to resolve the energy injection. The DNSs presented within capture the energy injection of both cases, as they have a triangular wavenumber truncation of $T=504$. This is equivalent to 1536 longitudinal and 768 latitudinal grid points.

It is found that for both the atmosphere and ocean, the SGS model coeff cients are approximately isotropic if the LES truncation wavenumber is signif cantly larger than $k_{R}$. The isotropised prof les decrease in magnitude and become steeper as resolution increases. A unif ed scaling law is determined that represents both the atmosphere and ocean SGS processes, by non-dimensionalising the model coeff cients on the basis of the f nal energy containing wavenumber $\left(k_{E}\right)$.
\end{abstract}

Keywords: Large Eddy Simulation, Atmosphere, Ocean, General Circulation Model, Stochastic Modelling 


\section{INTRODUCTION}

With the current level of computer hardware technology, it is not possible in a reasonable amount of time to simulate the atmosphere nor ocean by resolving all of the scales of motion. Instead one resorts to large eddy simulation (LES), where only the large-scales are resolved by a computational grid and the smaller scales are represented by a subgrid-scale (SGS) parameterisation. The present work is devoted to the development of such models, which are representative of the SGS processes in both the atmosphere and ocean. Typically the SGS processes are represented in an adhoc fashion, with tuning parameters manually adjusted for each simluation. Here, however, we adopt the SGS modelling approach of Frederiksen and Kepert (2006), where the model coeff cients are derived self-consistently from higher resolution direct numerical simulation (DNS). The stochastic SGS model consists of a backscatter noise term and a drain eddy viscosity. The aim of this study is to identify a set of universal scaling laws that represent the eddy viscosity and stochastic backscatter. This would remove the need to produce the high resolution DNS.

The paper is organised as follows. Section 2 presents a two-level quasi-geostrophic model, which is used to simulate the atmospheric and oceanic fows. These fow felds are discussed in section 3 . Section 4 presents how the SGS model coeff cients are determined from the DNS statistics, using the SGS modelling approach of Frederiksen and Kepert (2006). This approach is then applied to both the ocean and atmosphere data in section 5. The manner in which these model coeff cients change with resolution is identif ed, and the scaling laws are presented. Finally concluding remarks are made in section 6.

\section{QUASI-GEOSTROPHIC SPECTRAL EQUATIONS}

We employ the two-level quasi-geostrophic model of Frederiksen (1998). The numerical integration of which is an eff cient means of simulating geophysical fows, and captures the essential dynamics of baroclinic and barotropic instabilities. In the present study the vorticity is represented on two discrete vertical levels, with $j=1$ representing the upper level at $250 \mathrm{hPa}$, and $j=2$ the lower level at $750 \mathrm{hPa}$. The system is non-dimensionalised by using the radius of the earth $(a=6371 \mathrm{~km})$ as a length scale, and the inverse of the earth's angular velocity $\left(\Omega=7.292 \times 10^{-5} \mathrm{~s}^{-1}\right)$ as a time scale. By default all variables are assumed to be non-dimensional while the dimensional quantities are denoted by the $\backsim$ modif er.

The potential vorticity equation is spectrally discretised by expanding the feld variables in spherical harmonics with the zonal (longitudinal) wavenumber, $m$, and the total wavenumber, $n$. This results in the prognostic equations for the reduced potential vorticity spectral coeff cients, $q_{m n}^{j}=\zeta_{m n}^{j}+$ $(-1)^{j} F_{L}\left(\psi_{m n}^{1}-\psi_{m n}^{2}\right)$, where the superscript $j$ on the fow variables denotes the level, $F_{L}$ is the layer coupling parameter, $\zeta_{m n}^{j}=-n(n+1) \psi_{m n}^{j}$ are the spectral coeff cients of the vorticity, and $\psi_{m n}^{j}$ the streamfunction coeff cients. The evolution equation for $q_{m n}^{j}$ is

$$
\frac{\partial q_{m n}^{j}}{\partial t}=\mathrm{i} \sum_{p q} \sum_{r s} K_{n q s}^{m p r} \psi_{-p q}^{j} q_{-r s}^{j}-\mathrm{i} \omega_{m n} \zeta_{m n}^{j}-\alpha_{n}^{j} \zeta_{m n}^{j}+\kappa_{n}\left(\tilde{q}_{m n}^{j}-q_{m n}^{j}\right)-D_{0}^{j j}(n) q_{m n}^{j}
$$

where summations in (1) are over the triangular truncated wavenumber set

$$
\mathbf{T}=[p, q, r, s|-T \leq p \leq T,| p|\leq q \leq T,-T \leq r \leq T,| r \mid \leq s \leq T],
$$

with $T$ the DNS truncation wavenumber. The Rossby wave frequency is $\omega_{m n}=-B m /(n(n+1))$, where $B=2$ under the chosen scaling. The drag at each level is given by $\alpha_{n}^{j}$. The interaction coeff cients $K_{n q s}^{m p r}$ are detailed in Frederiksen and Kepert (2006). The model is driven toward the mean state $\tilde{q}_{m n}^{j}$, at a rate determined by the relaxation parameter $\kappa_{n}$. The bare dissipation is given by $D_{0}^{j l}(n)=\delta_{l j} D_{0}^{j j}(T)(n / T)^{\rho_{0}^{j}}$, where $\delta_{l j}$ is the Kronecker delta function, which ensures the off-diagonal elements of $D_{0}^{j l}(n)$ are zero. Here $D_{0}^{j j}(T)$ is the value of the diagonal elements at truncation and $\rho_{0}^{j}$ determines the steepness.

\section{CHARACTERISATION OF THE FLOW FIELDS}

We simulate typical large-scale atmospheric and oceanic fows. For the atmospheric cases the mean state $\tilde{q}_{m n}^{j}$ corresponds to large-scale westerly jets in the mid-latitudes of each hemisphere, and for the ocean 
$\tilde{q}_{m n}^{j}$ is representative of the Antarctic Circumpolar Current. In both cases the mean state is purely zonal with $\tilde{q}_{m n}^{j}=0$ unless $m=0$. For $m=0$ and $n \leq 15$ the relaxation time $\left(1 / \breve{\kappa}_{n}\right)$ is 11.6 days for the atmospheric simulations, and 116 days for the oceanic cases. For $m \neq 0$ or $n>15$ the relaxation parameter $\kappa_{n}=0$. Further details are presented on the atmospheric mean state in Zidikheri and Frederiksen (2009), and on the oceanic mean state in Zidikheri and Frederiksen (2010).

The other key difference between the atmospheric and oceanic simulations is the specif ed layer coupling parameter $F_{L}$. In the atmospheric cases $\vec{F}_{L}=2.5 \times 10^{-12} \mathrm{~m}^{-2}$, corresponding to a Rossby radius of deformation of $\breve{r}_{R} \equiv 1 / \sqrt{2 \breve{F}_{L}}=4.47 \times 10^{5} \mathrm{~m}$, and a non-dimensional Rossby wavenumber of $k_{R} \equiv$ $r_{R} / a \approx 14$. In the ocean simulations $\breve{F}_{L}=2.5 \times 10^{-10} \mathrm{~m}^{-2}$, with $\breve{r}_{R}=4.47 \times 10^{4} \mathrm{~m}$, and $k_{R} \approx 140$. Note $\breve{r}_{R}$ is an order of magnitude smaller in the ocean, which means that a f ner grid is required to capture the energy injection at the Rossby wavenumber.

In both cases a constant drag is applied to the energy containing scales $n \leq k_{E}$. For the atmosphere $k_{E}=14$, and the drag $\alpha_{n}^{j}$ has damping times $\left(1 / \breve{\alpha}_{n}^{j}\right)$ of 20 days for level 1 and 5 days for level 2 . For the ocean $k_{E}=50$, and the damping times are 40 days for level 1 and 10 days for level 2 .

The DNSs presented herein have a truncation wavenumber of $T=504$, and are denoted by $\mathcal{A} 504$ for the atmosphere and $\mathcal{O} 504$ for the ocean. This is equivalent to $1536 \times 768$ grid points (in longitude by latitude), or a resolution of 0.234 degrees, which along the equator corresponds to a grid point every $26 \mathrm{~km}$. At this resolution, the energy injection in both cases is resolved as $k_{R} \ll T$. The time step size is $\Delta \breve{t}=112 \mathrm{~s}$ for the atmosphere, and $\Delta \breve{t}=300 \mathrm{~s}$ for the ocean.

Instantaneous level 1 streamfunction felds minus the zonal component for the $\mathcal{A} 504$ and $\mathcal{O} 504$ DNS are shown in Fig. 1(a) and Fig. 1(b) respectively. The former illustrates dominant structures located in the mid-latitudes of the northern and southern hemispheres. The latter f gure illustrates dominant structures in the southern hemisphere, and these vortex structures are also smaller in size compared with the atmospheric case. This is consistent with the Rossby radius of deformation being an order of magnitude smaller in the ocean than in the atmosphere.

The dimensional total wavenumber $n$ kinetic energy spectra for level 1 and 2 are $\breve{e}_{1}(n)$ and $\breve{e}_{2}(n)$. Figure 2(a) shows these spectra for the $\mathcal{A} 504$ DNS. The $\breve{e}_{1}$ spectra is more energetic than $\breve{e}_{2}$, and for $n>k_{R}$ they both exhibit a constant enstrophy fux inertial range with an approximate $n^{-3}$ power law. This is consistent with the theoretical power law for two-dimensional turbulence of Kraichnan (1967). The enstrophy f uxes within this region are $\breve{\eta}_{1}=1.2 \times 10^{-15} \mathrm{~s}^{-3}$ and $\breve{\eta}_{2}=4.3 \times 10^{-16} \mathrm{~s}^{-3}$ for levels 1 and 2 respectively. In non-dimensional form $\eta_{1} \equiv \breve{\eta}_{1} \Omega^{3}=3.1 \times 10^{-3}$ and $\eta_{2} \equiv \breve{\eta}_{2} \Omega^{3}=1.1 \times 10^{-3}$. Figure 2(b) illustrates the spectra for the $\mathcal{O} 504$ DNS. These spectra are less energetic in the energy containing scales as compared to the atmosphere, but still exhibit an approximate $n^{-3}$ power law for $n>k_{R}$. The ocean simulations also exhibits an approximate $n^{-\frac{5}{3}}$ slope within the wavenumber range $k_{E}<n<k_{R}$ con-

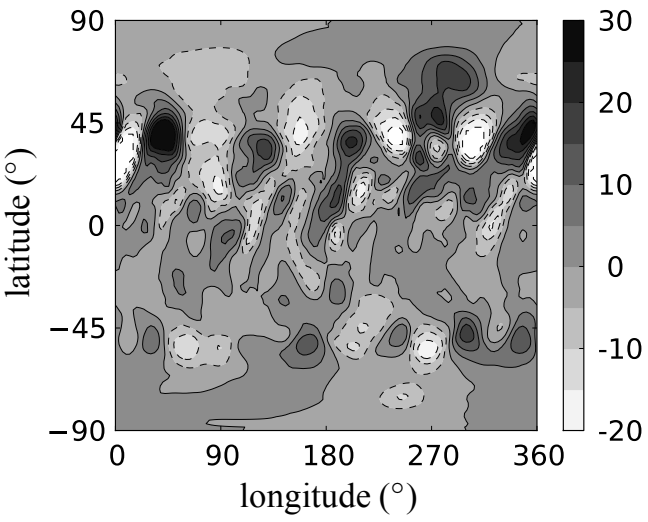

(a)

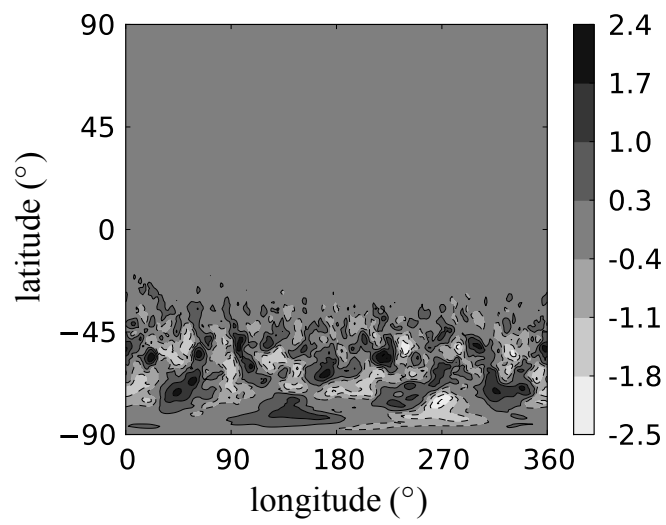

(b)

Figure 1. The upper level streamfunction feld minus the zonal component for the: (a) $\mathcal{A} 504$ DNS; and (b) $\mathcal{O} 504$ DNS. The colour bars have units of $\mathrm{s}^{-1}$. 
sistent with an inverse energy cascade (Kraichnan, 1967). The constant enstrophy f uxes within the range $n>k_{R}$ are $\breve{\eta}_{1}=1.8 \times 10^{-15} \mathrm{~s}^{-3}$ and $\breve{\eta}_{2}=1.4 \times 10^{-15} \mathrm{~s}^{-3}$; and in non-dimensional form $\eta_{1}=4.9 \times 10^{-3}$ and $\eta_{2}=3.6 \times 10^{-3}$. These enstrophy f uxes are important because Leith (1971) showed that the eddy viscosity in the enstrophy cascading inertial range is proportional to $\eta_{j}^{1 / 3}$.

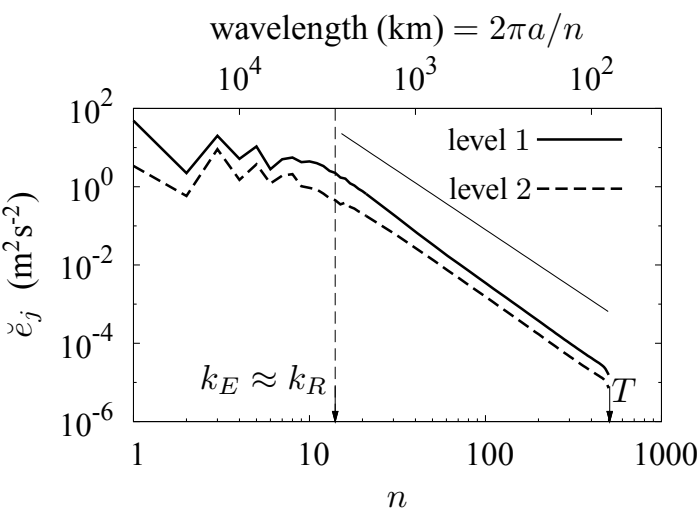

(a)

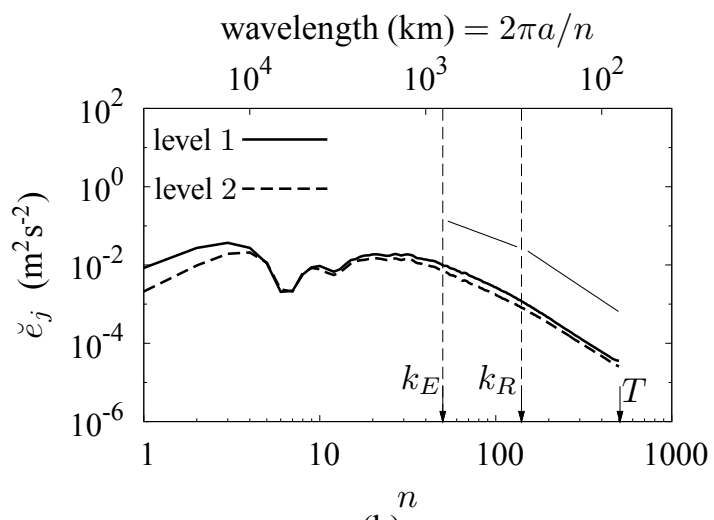

(b)

Figure 2. The kinetic energy spectra for the: (a) $\mathcal{A} 504$ DNS, with the reference line $n^{-3}$ for $k_{R}<n$; and (b) $\mathcal{O} 504$ DNS, with the reference lines $n^{-5 / 3}$ for $k_{E}<n<k_{R}$, and $n^{-3}$ for $k_{R}<n$.

\section{STOCHASTIC SUBGRID SCALE MODEL}

We now apply the stochastic modelling approach of Frederiksen and Kepert (2006) to parameterise the effects of the subgrid-scale eddies on the resolved scales in LESs. In LESs the resolution is reduced compared with DNS, and conf ned to the set $\mathbf{R}$ with a LES truncation wavenumber $T_{R}<T$. The subgrid wavenumber set is def ned as $\mathbf{S}=\mathbf{T}-\mathbf{R}$. To facilitate a discussion on this decomposition of the fow, we let $\mathbf{q}$ equal the transpose of $\left(q_{m n}^{1}, q_{m n}^{2}\right)$ for a given wavenumber pair. In this vector notation $\mathbf{q}_{t}(t)=\mathbf{q}_{t}^{\mathbf{R}}(t)+\mathbf{q}_{t}^{\mathbf{S}}(t)$, where $\mathbf{q}_{t}$ is the tendency (or time derivative) of $\mathbf{q}$. The tendency of the resolved scales is $\mathbf{q}_{t}^{\mathbf{R}}$, where all triadic interactions involve wavenumbers less than $T_{R}$. The remainder is the subgrid tendency $\mathbf{q}_{t}^{\mathbf{S}}$, for which at least one wavenumber component involved in the triadic interactions is greater than $T_{R}$. The subgrid tendency is further decomposed into a time averaged $\overline{\mathbf{f}} \equiv \overline{\mathbf{q}_{t}^{\mathbf{S}}}$ and a f uctuating component $\widehat{\mathbf{q}}_{t}^{\mathrm{S}}$. We use the values of $\overline{\mathbf{f}}$ taken from the DNS, and $\widehat{\mathbf{q}}_{t}^{\mathbf{S}}$ is modelled.

The f uctuating component of the subgrid tendency is represented by the stochastic equation

$$
\widehat{\mathbf{q}}_{t}^{\mathbf{S}}(t)=-\mathbf{D}_{\mathbf{d}} \widehat{\mathbf{q}}(t)+\widehat{\mathbf{f}}(t),
$$

where $\mathbf{D}_{\mathbf{d}}$ is the subgrid drain dissipation matrix, $\widehat{\mathbf{q}}$ is the fuctuating component of $\mathbf{q}$, and $\widehat{\mathbf{f}}$ is a random forcing vector. The drain $\mathbf{D}_{\mathbf{d}}$ is determined by post-multiplying both sides of (3) by $\widehat{\mathbf{q}}^{\dagger}\left(t_{0}\right)$, integrating over the decorrelation period $\tau$, and ensemble averaging to produce the least squares approximation

$$
\mathbf{D}_{\mathbf{d}}=-\left\langle\int_{t_{0}}^{t} \widehat{\mathbf{q}}_{t}^{\mathbf{S}}(\sigma) \widehat{\mathbf{q}}^{\dagger}\left(t_{0}\right) d \sigma\right\rangle\left\langle\int_{t_{0}}^{t} \widehat{\mathbf{q}}(\sigma) \widehat{\mathbf{q}}^{\dagger}\left(t_{0}\right) d \sigma\right\rangle^{-1},
$$

where $\dagger$ denotes the Hermitian conjugate for vectors and matrices. The angled brackets denote ensemble averaging, with each ensemble member determined by shifting the initial time $t_{0}$ and the f nal time $t=$ $t_{0}+\tau$ forward by one time step. The drain eddy viscosity is given by $\boldsymbol{\nu}_{\mathbf{d}}=\mathbf{D}_{\mathbf{d}} / n /(n+1)$.

The model for $\widehat{\mathbf{f}}$ is determined by calculating the nonlinear noise covariance matrix $\mathcal{F}_{\mathbf{b}}=\mathbf{F}_{\mathbf{b}}+\mathbf{F}_{\mathbf{b}}{ }^{\dagger}$, where $\mathbf{F}_{\mathbf{b}}=\left\langle\widehat{\mathbf{f}}(t) \widehat{\mathbf{q}}^{\dagger}(t)\right\rangle$. By post-multiplying both sides of (3) by $\widehat{\mathbf{q}}^{\dagger}(t)$, and adding the conjugate transpose of (3) pre-multiplied by $\widehat{\mathbf{q}}(t)$ yields the Lyapunov or balance equation

$$
\left\langle\widehat{\mathbf{q}}_{t}^{\mathbf{S}}(t) \widehat{\mathbf{q}}^{\dagger}(t)\right\rangle+\left\langle\widehat{\mathbf{q}}(t) \widehat{\mathbf{q}}_{t}^{\mathbf{S}}(t)\right\rangle=-\mathbf{D}_{\mathbf{d}}\left\langle\widehat{\mathbf{q}}(t) \widehat{\mathbf{q}}^{\dagger}(t)\right\rangle-\left\langle\widehat{\mathbf{q}}(t) \widehat{\mathbf{q}}^{\dagger}(t)\right\rangle \mathbf{D}_{\mathbf{d}}^{\dagger}+\mathcal{F}_{\mathbf{b}} .
$$


Given that $\mathbf{D}_{\mathbf{d}}$ is known, $\mathcal{F}_{\mathbf{b}}$ can now be calculated. The formulation is general, and $\widehat{\mathbf{f}}$ is calculated based on being coloured noise. After calculation of $\mathcal{F}_{\mathbf{b}}$, however, we f nd that it is suff cient to model $\widehat{\mathbf{f}}$ as the white noise process $\left\langle\widehat{\mathbf{f}}(t) \widehat{\mathbf{f}}^{\dagger}\left(t^{\prime}\right)\right\rangle=\mathcal{F}_{\mathbf{b}} \delta\left(t-t^{\prime}\right)$. To represent the backscatter in a similar manner to the drain, the backscatter eddy viscosity is given by $\boldsymbol{\nu}_{\mathbf{b}}=\mathbf{D}_{\mathbf{b}} / n /(n+1)=-\mathbf{F}_{\mathbf{b}}\left\langle\widehat{\mathbf{q}}(t) \widehat{\mathbf{q}}^{\dagger}(t)\right\rangle^{-1} / n /(n+1)$.

The evolution equation for $q_{m n}^{j}$ in the LES is equivalent to that of the DNS in (1), with the addition of the SGS tendency $\left(q_{t}^{S}\right)_{m n}^{j}$ to the right-hand-side, and solved over the wavenumber set $\mathbf{R}$ instead of $\mathbf{T}$. The SGS tendency is represented using (3).

\section{EDDY VISCOSITY RESULTS}

The SGS model coeff cients are now determined from the statistics of the DNS using the approach outlined in the previous section. Firstly for the atmospheric case, the $\mathcal{A} 504$ DNS is truncated back to an LES truncation wavenumber of $T_{R}=63$. For each wavenumber pair all of the required coeff cients are determined, with the upper diagonal component of the drain eddy viscosity $\nu_{d}^{11}$, illustrated in Fig. 3(a). These coeff cients are positive, increase according to a power law in $n$, and for a given $n$ are weakly dependent upon $m$. The $\nu_{d}^{22}$ component has a similar structure, and the off-diagonal terms $\nu_{d}^{12}$ and $\nu_{d}^{21}$, are negligible in comparison. The drain eddy viscosity is isotropised by averaging over $m$ and plotted against $n$ in Fig. 3(c). This clearly illustrates a cusp-like form. The truncation procedure is repeated for $T_{R}=126$ and $T_{R}=252$, with the isotropised prof les also included in Fig. 3(c). A comparison of these prof les identif es that as the LES resolution increases the maximum value of the drain prof les decreases. The backscatter coeff cients also have similar properties to $\nu_{\mathbf{d}}$.

Likewise the $\mathcal{O} 504$ DNS is truncated back to $T_{R}=63$, with the $\nu_{d}^{11}$ component illustrated in Fig. 3(b). As in the atmospheric case, these coeff cients increase with $n$, but here have a signif cant dependence upon $m$. Some of the coeff cients centred at $(m, n)=(15,20)$ are also negative. The $\nu_{d}^{22}$ component also has a similar structure to $\nu_{d}^{11}$. In this case the off-diagonal terms are signif cant, and mainly negative throughout the $(m, n)$ wavenumber plane. The truncation is repeated for $T_{R}=126$ and $T_{R}=252$, with the isotropised prof les plotted in Fig. 3(d). Consistent with the observations of the atmospheric results, as the LES resolution increases the maximum value of the drain prof les decrease. In addition, as the resolution increases, the negative component disappears, and the off-diagonal terms become less signif cant.

For both the ocean and atmosphere simulations, the wavenumber range over which the eddy viscosities are non-zero is related to the last energy containing wavenumber $k_{E}$. Kraichnan (1976) states that the signif cantly non-zero values of the eddy viscosity are concentrated among the last $k_{E}$ wavenumbers before truncation. This means that SGS nonlinear interactions are signif cant within the range $T_{R}-k_{E} \lesssim$ $n \leq T_{R}$. We are primarily concerned with the approximate lower bound $T_{R}-k_{E}$. Recall that for the $\mathcal{A} 504$ DNS the last of the energy containing scales occurs at $k_{E} \approx 14$, and the Rossby wavenumber $k_{R} \approx 14$. For all of the atmospheric truncations in this study $T_{R}-k_{E}>k_{R}$, which means the SGS nonlinear interactions are conf ned to within the self-similar enstrophy cascading range. This is not the case for all of the ocean truncation levels. In the $\mathcal{O} 504$ DNS $k_{E} \approx 50$, and $k_{R} \approx 140$. For the $T_{R}=252$ case, $T_{R}-k_{E}>k_{R}$ and the SGS nonlinear interactions are again conf ned to within the region of constant enstrophy f ux. This explains why the isotropic prof le of the $T_{R}=252$ ocean case in Fig. 3(d), qualitatively resembles the atmospheric prof les in Fig. 3(c). The eddy viscosity prof le for the $T_{R}=126$ ocean case has a different structure because $T_{R}-k_{E}<k_{R}$. This means there are signif cant SGS nonlinear interactions within the wavenumber region $k_{E}<n<k_{R}$, and here the enstrophy $\mathrm{f} u x$ is not constant. The physics within this region, however, are still self similar and more complicated scaling laws could possibly be developed for truncations made within this wavenumber range. The coeff cients for the $T_{R}=63$ ocean case have a different structure again because $T_{R}-k_{E}<k_{E}$, which means there are signif cant interactions with the energy containing scales themselves. It is unlikely that scaling laws could be developed for these truncation levels as the energy containing scales are problem specif c.

The diagonal components of the isotropic drain eddy viscosity prof les are characterised by least squares f tting them to the power law $\nu_{d}^{j j}(n)=\nu_{d}^{j j}\left(T_{R}\right)\left(n / T_{R}\right)^{\rho_{d}^{j}}$, where $\nu_{d}^{j j}\left(T_{R}\right)$ is the value at truncation on level $j$. The parameter $\rho_{d}^{j}$ controls the steepness of the prof le, and hence the width of the non-zero eddy viscosity region in wavenumber space. Since the width of this region is proportional to $k_{E}, \rho_{d}^{j}$ is plotted 


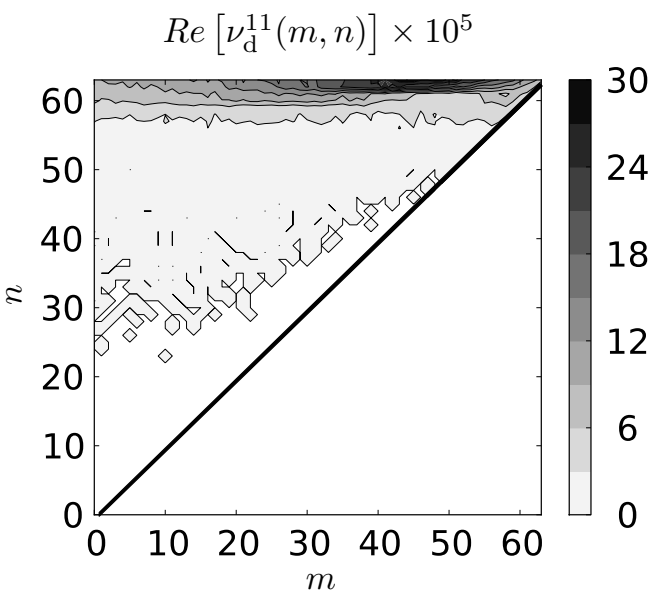

(a)

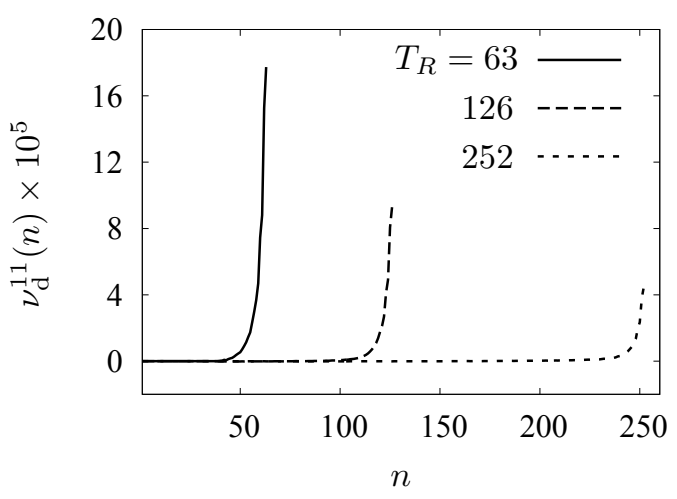

(c)

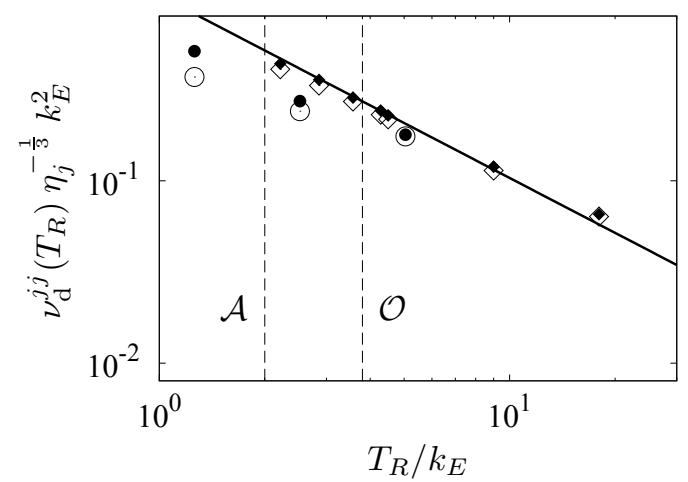

(e)

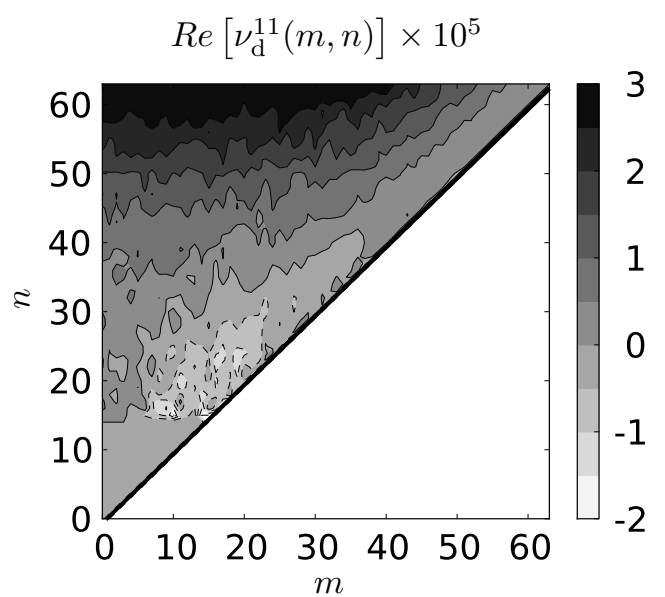

(b)

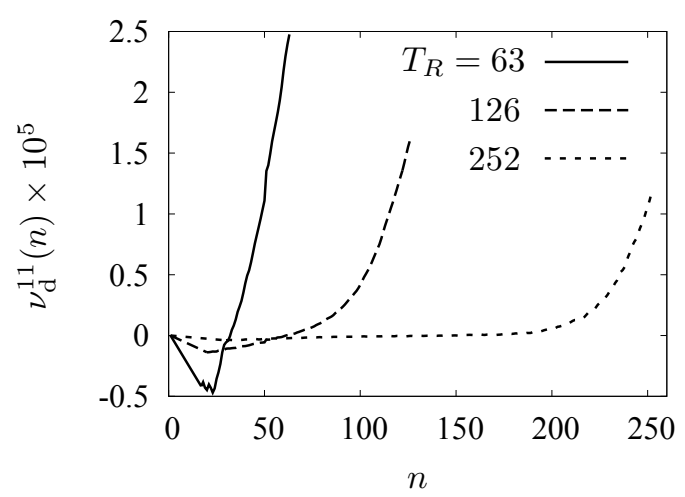

(d)

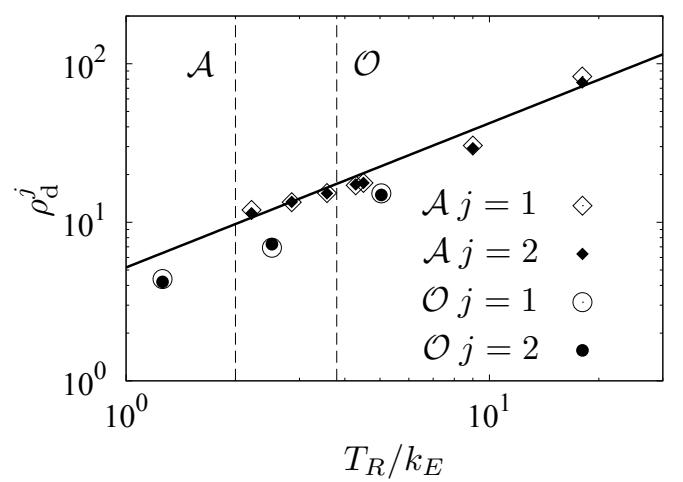

(f)

Figure 3. Drain eddy viscosity anisotropic coeff cient $\nu_{\mathrm{d}}^{11}(m, n)$ for the: (a) $\mathcal{A} 504$; and (b) $\mathcal{O} 504 \mathrm{DNS}$, both truncated back to $T_{R}=63$. Isotropic coeff cient $\nu_{\mathrm{d}}^{11}(n)$ for the: (c) $\mathcal{A} 504$; and (d) $\mathcal{O} 504 \mathrm{DNS}$, both truncated back to $T_{R}=63,126$, and 252. Isotropic scaling laws for the: (e) maximum value $\nu_{\mathrm{d}}^{j j}\left(T_{R}\right)$; and (f) power exponent $\rho_{\mathrm{d}}^{j}$, of the diagonal components with the least squares line $\mathrm{ft}$ only to the atmospheric data points. Note the key in (f) also represents the data in (e). The vertical lines within (e) and (f), represent estimated truncation thresholds $T_{R} / k_{E}=\left(k_{R}+k_{E}\right) / k_{E}$, above which all signif cant SGS nonlinear interactions occur within the constant enstrophy cascading range, and the labels $\mathcal{A}$ and $\mathcal{O}$ representing the atmospheric and oceanic thresholds respectively. 
against $T_{R}$ non-dimensionalised by $k_{E}$ in Fig. 3(f). The diamond symbols represent the atmospheric data, circles the ocean, the hollow symbols are for level $j=1$, and flled symbols for $j=2$. The dashed vertical lines represent the estimated truncation thresholds above which all signif cant SGS nonlinear interactions occur within the constant enstrophy cascading range; that is $T_{R} / k_{E}=\left(k_{R}+k_{E}\right) / k_{E}$, with the labels $\mathcal{A}$ and $\mathcal{O}$ representing to atmospheric and oceanic thresholds respectively. Note all of the atmospheric LESs are greater than this threshold; however, this is not the case for the ocean. A trend line is, therefore, only $\mathrm{f}$ tted to the atmospheric data producing the equations

$$
\rho_{d}^{j}=5.2\left(T_{R} / k_{E}\right)^{0.91}, \text { and } \rho_{b}^{j}=9.1\left(T_{R} / k_{E}\right)^{0.91},
$$

where $\rho_{b}^{j}$ is the analogous power exponent for the backscatter eddy viscosity $\nu_{\mathbf{b}}$. Only the $T_{R}=252$ ocean case is in the enstrophy cascading range, and its value of $\rho_{d}^{j}$ in (6) is consistent with the atmospheric data. The maximum value $\nu_{d}^{j j}\left(T_{R}\right)$ is plotted in Fig. 3(e) scaled by $\eta_{j}^{-1 / 3}$ to collapse the data between levels (see section 3), and non-dimensionalised by $k_{E}$ to collapse the atmospheric and oceanic data. A trend line is $\mathrm{ftted}$ to the atmospheric data, producing

$$
\nu_{d}^{j j}\left(T_{R}\right)=0.88\left(T_{R} / k_{E}\right)^{-1}, \text { and } \nu_{b}^{j j}\left(T_{R}\right)=-0.43\left(T_{R} / k_{E}\right)^{-1},
$$

where $\nu_{b}^{j j}\left(T_{R}\right)$ is the value at trunction for $\nu_{\mathbf{b}}$. The $T_{R}=252$ ocean points agrees with the atmospheric data, and the lower resolution ocean cases do not. The lower resolution oceanic LES data points also begin to separate between levels, which indicates that the enstrophy fux scaling does not hold outside of the constant enstrophy f ux region. In Kitsios et al. (2011) these scaling laws are used for the LES of atmospheric fows, and are shown to replicate the kinetic energy spectra of DNS.

\section{CONCLUding REMARKS}

The scaling laws presented within, make the SGS parameterisations more generally applicable in LESs as they remove the need to generate the model coeff cients from a high resolution DNS. Scaling laws for $\nu_{\mathrm{d}}$ have been made applicable to simulations of both the atmosphere and ocean by non-dimensionalising them on the basis of the last energy containing wavenumber $k_{E}$. The scaling laws are currently valid for LES truncations made within the constant enstrophy cascading range, such that $T_{R}>k_{R}+k_{E}$. Additional scaling laws are currently being developed for LES truncations made within the range $k_{E}<T_{R}<k_{R}$.

\section{ACKNOWLEDGEMENT}

V. Kitsios acknowledges the CSIRO Off ce of the Chief Executive for funding his post-doctoral position.

\section{REFERENCES}

Frederiksen, J. S. (1998). Precursors to blocking anomalies: the tangent linear and inverse problems. J. Atmos. Sci. 55, 2419-2436.

Frederiksen, J. S. and S. M. Kepert (2006). Dynamical subgrid-scale parameterizations from Direct Numerical Simulations. J. Atmos. Sci. 63, 3006-3019.

Kitsios, V., J. Frederiksen, and M. Zidikheri (2011). Subgrid model with scaling laws for atmospheric simulations. under review.

Kraichnan, R. (1967). Inertial ranges in two-dimensional turbulence. Phys. Fluids 10(7), 1417-1423.

Kraichnan, R. (1976). Eddy viscosity in two and three dimensions. J. Atmos. Sci. 33, 1521-1536.

Leith, C. E. (1971). Atmospheric predictability and two-dimensional turbulence. J. Atmos. Sci. 28, 145 161.

Zidikheri, M. J. and J. S. Frederiksen (2009). Stochastic subgrid parameterizations for simulations of atmospheric baroclinic fows. J. Atmos. Sci. 66, 2844-2858.

Zidikheri, M. J. and J. S. Frederiksen (2010). Stochastic subgrid-sacle modelling for non-equilibrium geophysical f ows. Phil. Trans. Royal Soc. A 368, 145-160. 